\title{
Modeling COVID-19 Data with a Novel Extended Exponentiated Class of Distributions
}

\author{
Muhammad Arif, ${ }^{1}$ Dost Muhammad Khan ${ }^{(D)},{ }^{1}$ Muhammad Aamir ${ }^{(D)},{ }^{1}$ Umair Khalil, ${ }^{1}$ \\ Rashad A. R. Bantan, ${ }^{2}$ and Mohammed Elgarhy ${ }^{3}{ }^{3}$ \\ ${ }^{1}$ Department of Statistics, Abdul Wali Khan University Mardan, KP, Pakistan \\ ${ }^{2}$ Department of Marine Geology, Faculty of Marine Science, King Abdul Aziz University, Jeddah 21551, Saudi Arabia \\ ${ }^{3}$ The Higher Institute of Commercial Sciences, Al Mahalla Al Kubra, Algarbia 31951, Egypt \\ Correspondence should be addressed to Mohammed Elgarhy; m_elgarhy85@sva.edu.eg
}

Received 8 November 2021; Revised 14 December 2021; Accepted 31 December 2021; Published 23 February 2022

Academic Editor: Gul Rahmat

Copyright (c) 2022 Muhammad Arif et al. This is an open access article distributed under the Creative Commons Attribution License, which permits unrestricted use, distribution, and reproduction in any medium, provided the original work is properly cited.

\begin{abstract}
The COVID-19 epidemic has affected every aspect of daily life since December 2019 and caused massive damage to the world. The coronavirus epidemic has affected more than 150 countries around the world. Many researchers have tried to develop a statistical model which can be utilized to analyze the behavior of the COVID-19 data. This article contributes to the field of probability theory by introducing a novel family of distributions, named the novel extended exponentiated class of distributions. Explicit expressions for numerous mathematical characterizations of the proposed family have been obtained with special concentration on a three-parameter submodel of the new class of distributions, named the new extended exponentiated Weibull distribution. The unknown model parameter estimates are obtained via the maximum likelihood estimation method. To assess the performance of these estimates, a comprehensive simulation study is conducted. Three different sets of COVID-19 data are used to check the applicability of the submodel case. The submodel of the new family is compared with three well-known probability distributions. Using different analytical measures, the results demonstrate that the new extended exponentiated Weibull distribution gives promising results in terms of its flexibility and offers data modeling with increasing decreasing, unimodal, and modified unimodal shapes.
\end{abstract}

\section{Introduction}

Probability distributions play a vital role in predicting and defining real-world phenomena. While modeling lifetime data, most researchers use some well-known existing distributions like beta, Rayleigh, Weibull, and exponential distributions, but some of these existing probability models have certain limitations. For instance, the exponential model provides only constant hazard rate shapes, and the Rayleigh distribution exhibits an increasing hazard rate while modeling lifetime data. Whereas the cumulative distribution function (CDF) of beta and gamma distributions does not exist in closed form, which creates problems in estimating parameters. However, the Weibull model is the most prominent model used to model data having constant, decreasing, and increasing behavior, but is not suitable for nonmonotonic (unimodal, modified unimodal, or bathtub) hazard rates. In real-life situations, many mechanical and electronic components have increasing and unimodal hazard (failure) rate functions. For example, the failure rate of an electronic product may be higher after its fifteen years of service than the failure rate during the initial years. Similarly, in the medical sector, the risk rate of bladder cancer relapsing after surgery has been noticed to be unimodal in nature. Initially, the hazard rate for breast cancer recurrence usually occurs at the lowest point, steadily rises to a peak, then starts decreasing after surgical removal. Therefore, to model such behaviors of data, different distributions have been proposed, modified, and extended the existing distribution by various researchers in the literature. 
However, many authors have exhibited a deep interest in offering new distributional families in recent literature. An attractive idea for extension of the existing distributions in the literature is provided to introduce a new family of distributions using logit function (see [1] for more details), similarly the gamma-X family of distributions by [2] and T-X family of distributions by [3] are the major contributions to the probability theory. More data about recent families of distributionsare given in [4-7].

Recently, a well-known approach in this domain is given by [8], called, alpha power transformation (APT). The CDF of the APT class of distributions is given as follows:

$$
G_{\mathrm{APT}}(x ; \alpha, \xi)= \begin{cases}\frac{\alpha^{F(x ; \xi)}-1}{\alpha-1}, & \text { if } \alpha>0, \alpha \neq 1 . \\ F(x ; \xi), & \text { if } \alpha=1 .\end{cases}
$$

They presented a submodel case of the proposed family by using one parameter exponential distribution, named the alpha power exponential distribution. Since then, using the APT technique, various lifetime models have been developed (see [9-12]). Some well-known probability models that contributed to the probability theory using COVID-19 data are considered by [13-16]. Similarly, some other well-known probability models were introduced by different authors for modeling COVID-19 data. They considered different COVID-19 datasets from different countries (see [17-20] for more details).

Another well-known technique, named the quadratic rank transmutation (QRT) technique considered by [21]. The CDF of the QRT method is presented as follows :

$$
\begin{gathered}
G(x ; \lambda, \xi)=(1+\lambda) F(x ; \xi)-\lambda F(x ; \xi)^{2}, \\
\xi>0, x \in \mathbb{R},|\lambda| \leq 1,
\end{gathered}
$$

where, $\lambda$ is known as a transmuted parameter. Many authors have considered the transmuted forms of the existing distributions with real data applications using equation (2). For more detail, the interested readers can refer to [22-24].

Recently a new contribution has been made to the probability theory by analyzing a new generalized class of distributions called, the Kumaraswamy G (Ku-G) family (see [25] for more details). The expression for CDF is provided as

$$
G(x ; \alpha, b, \xi)=1-\left[1-F(x ; \xi)^{\alpha}\right]^{b}, \quad \alpha, b, \xi>0, x \in R,
$$

where, $\alpha>0$ and $b>0$ denoting two additional shape parameters. Recently, researchers have introduced novel families of probability distributions and analyzed COVID19 data through the suggested models. They presented different plots of the fitted data and compared the suggested models with the other existing probability models (see $[26,27]$ for more details). Furthermore, some authors contributed to the probability theory by introducing new models (see $[28,29]$ ).

However, these distributions have been shown to have less flexibility and a lack of fit in different situations for different real datasets. Moreover, there are some distributions in the literature that do not have a closed-form CDF. The main weakness of many distributions is that they cannot fit the modified unimodal shapes. Similarly, some authors introduced new families by adding two or more extra parameters, which causes difficulties in the estimation of the unknown parameters.

Therefore, this paper attempts to capitalize on a novel state-of-the-art procedure that encompasses all the weaknesses of the available methods in terms of flexibility and lack of fit for different datasets. A special submodal case of the NEE class exhibits that the newly proposed family can model the data having unimodal, decreasing, modified unimodal, and increasing shapes of hazard rates.

Moreover, the suggested family of distributions is very simple and flexible as compared to the existing families of distributions.

(i) the simplest and most versatile method to adjust an extra parameter for modification of existing probability distributions;

(ii) to deliver greater enhancement in the characterizations and to make the current probability models more flexible;

(iii) to present the new extended form of the existing model, comprising closed-form CDF and hrf;

(iv) generalization of the current probability models by incorporating only a single parameter instead of more parameters;

(v) to deliver the model, offering superior fits than the other compared models available in the literature.

The remaining part of the paper is structured as follows: Section 2 contains the introduction of the newly proposed family. The general form of the CDF, probability density function (pdf), survival function, and hazard function are provided in this section. The special case of the NEE class is provided in Section 3. Mathematical characterizations that include raw moments, quantile functions, and momentgenerating functions are addressed in Section 4. Furthermore, Section 5 discusses the parameter estimation and simulation analysis of the suggested model. The simulation results, along with their graphical display, are provided in this section. The applications of three real-life datasets are discussed in Section 6. This section contains the three different COVID-19 datasets, which show the applicability and model adequacy of the proposed model. Section 7 provides the limitations of the suggested model, while the future research direction is provided in Section 8. Finally, the conclusion of the article is provided in Section 9.

\section{Novel Extended Exponentiated Class of Distributions}

This article introduces a new family of continuous distributions, named the novel extended exponentiated (NEE) class of distributions. The general form of the CDF of the new family is presented as follows: 


$$
G(x ; \theta, \xi)=\frac{\left(e^{\theta F(x ; \xi)}+1\right)(F(x ; \xi))^{2}}{e^{\theta}+1} .
$$

However, $F(x ; \xi)$ represents the CDF of the existing probability distribution having a vector parameter $\xi$, while $\alpha$ is an extra parameter. The expressions for the pdf, survival function, and hazard function of the corresponding CDF given by equation (4), are provided respectively.

$$
\begin{aligned}
& g(x ; \alpha, \xi)=\frac{f(x ; \xi) F(x ; \xi)\left\{e^{\theta F(x ; \xi)}(2+\theta F(x ; \xi))+2\right\}}{e^{\theta}+1}, \\
& S(x ; \alpha, \xi)=1-\frac{\left(e^{\theta F(x ; \xi)}+1\right)(F(x ; \xi))^{2}}{e^{\theta}+1}, \\
& h(x ; \alpha, \xi)=\frac{f(x ; \xi) F(x ; \xi)\left\{e^{\theta F(x ; \xi)}(2+\theta F(x ; \xi))+2\right\}}{\left(e^{\theta}+1\right)-\left\{e^{\theta F(x ; \xi)}+1\right\}(F(x ; \xi))^{2}} .
\end{aligned}
$$

\section{New Extended Exponentiated Weibull Model}

This part of the article offers a distinctive submodel case of the NEE class, called the new extended exponentiated Weibull (NEE-W) distribution.

Let $F(x ; \xi)$ and $f(x ; \xi)$ representing the CDF and pdf of the two-parameter Weibull model, then the expression for the CDF of NEE-W is

$$
G(x ; \alpha, \lambda, \theta)=\frac{\left(e^{\theta\left(1-e^{-\alpha x^{\lambda}}\right)}+1\right)\left(1-e^{-\alpha x^{\lambda}}\right)^{2}}{e^{\theta}+1}, \quad \alpha, \lambda, \theta>0, x \geq 0 .
$$

The expressions for the pdf, survival, and hazard functions, respectively, are given as follows:

$$
\begin{aligned}
& g(x ; \alpha, \lambda, \theta)=\frac{\alpha \lambda x^{\lambda-1} e^{-\alpha x^{\lambda}}\left(1-e^{-\alpha x^{\lambda}}\right)\left\{e^{\theta\left(1-e^{-\alpha x^{\lambda}}\right)}\left(2+\theta\left(1-e^{-\alpha x^{\lambda}}\right)\right)+2\right\}}{e^{\theta}+1}, \quad x \geq 0, \\
& S(x ; \alpha, \lambda, \theta)=1-\frac{\left(e^{\theta\left(1-e^{-\alpha x^{\lambda}}\right)}+1\right)\left(1-e^{-\alpha x^{\lambda}}\right)^{2}}{e^{\theta}+1}, \quad x \geq 0, \\
& h(x ; \alpha, \lambda, \theta)=\frac{\alpha \lambda x^{\lambda-1} e^{-\alpha x^{\lambda}}\left(1-e^{-\alpha x^{\lambda}}\right)\left\{e^{\theta\left(1-e^{-\alpha x^{\lambda}}\right)}\left(2+\theta\left(1-e^{-\alpha x^{\lambda}}\right)\right)+2\right\}}{e^{\theta}+1-\left(e^{\theta\left(1-e^{-\alpha x^{\lambda}}\right)}+1\right)\left(1-e^{-\alpha x^{\lambda}}\right)^{2}}, \quad x \geq 0 .
\end{aligned}
$$

The plots presented in Figure 1 show different behaviors of the NEE-W model. The density of the NEE-W model enjoys symmetrical and asymmetrical shapes, including positively skewed, negatively skewed, and reversed J shapes. Figure 2 exhibits that the NEE-W model can model modified unimodal, increasing, decreasing, and upside-down bathtub-shaped failure rates.

\section{Mathematical Properties}

This section article provides the basic mathematical characterizations including, raw moments, quantile function, and moment generating function of the NEE class of distributions.

4.1. Raw Moments. In statistical analysis, the role of moments is very crucial in determining the key features and important characterizations of the model. The expressions for the $r^{\text {th }}$ order moments of the NEE class of distributions are derived as

$$
\begin{aligned}
g(x ; \theta, \xi) & =\frac{f(x ; \xi) F(x ; \xi)\left\{2+2 e^{\theta F(x ; \xi)}+\theta F(x ; \xi) e^{\theta F(x ; \xi)}\right\}}{e^{\theta}+1}, \\
\mu_{r}^{\prime} & =\int_{\Omega} x^{r} g(x ; \theta, \xi) \mathrm{d} x, \\
\mu_{r}^{\prime} & =\int_{\Omega} \frac{x^{r} f(x ; \xi) F(x ; \xi)\left\{2+2 e^{\theta F(x ; \xi)}+\theta F(x ; \xi) e^{\theta F(x ; \xi)}\right\}}{e^{\theta}+1} \mathrm{~d} x .
\end{aligned}
$$




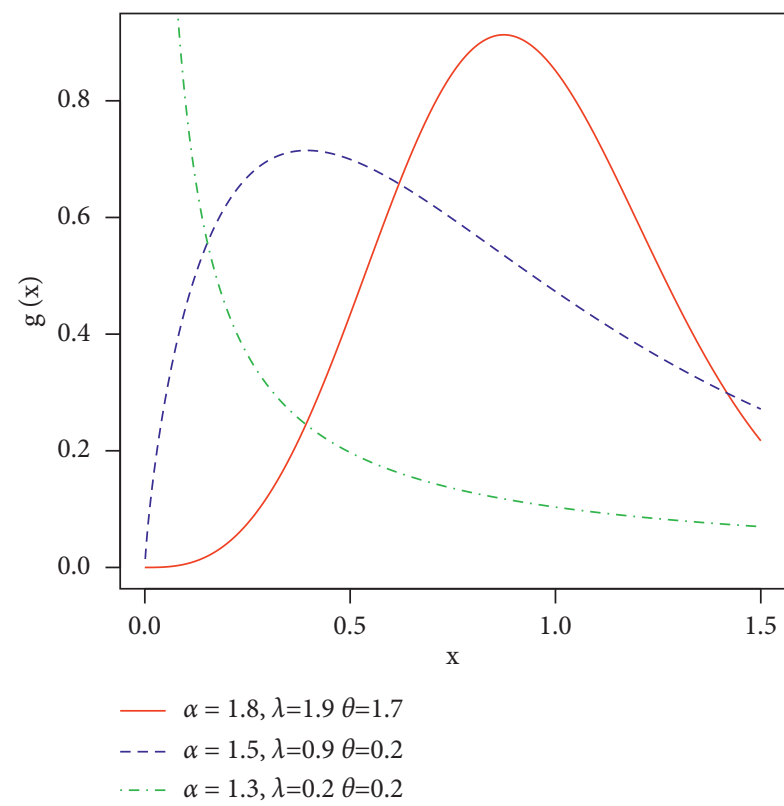

(a)



(b)

Figure 1: Plots of the pdf using different values of parameters.

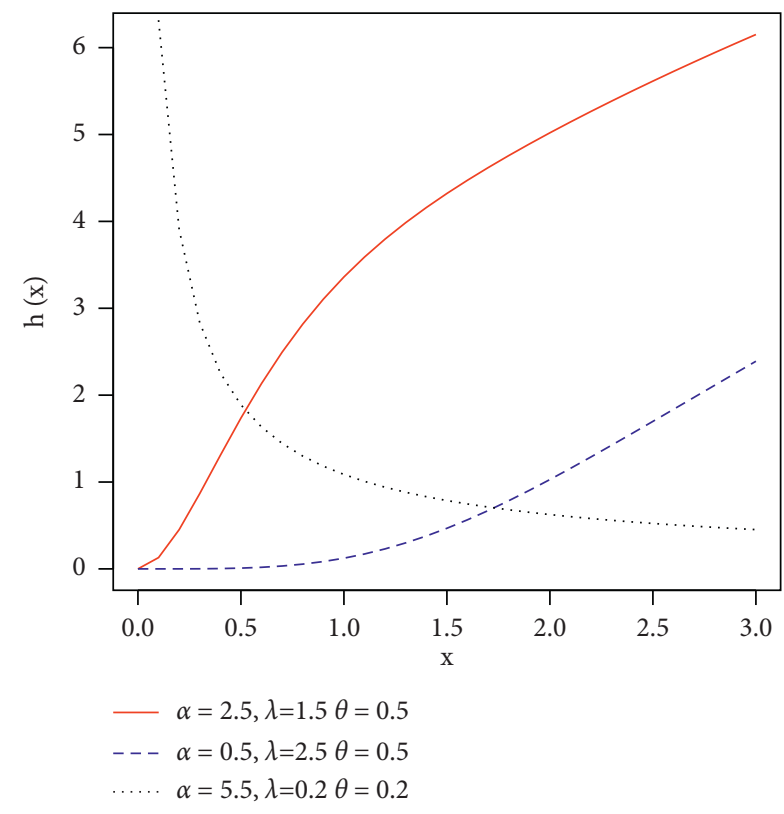

(a)



(b)

FIgURe 2: Plots of hazard functions for different values of parameters. 
As we know that

Using equation (9), we have

$$
e^{x}=\sum_{n=0}^{\infty} \frac{x^{n}}{n !}, \text { Let } x=\theta F(x ; \xi) .
$$

$$
\begin{gathered}
\mu_{r}^{\prime}=\frac{1}{\left(e^{\theta}+1\right)} \int_{\Omega} x^{r} f(x ; \xi) F(x ; \xi)\left\{2+2 \sum_{n=0}^{\infty} \frac{\theta^{n}(F(x ; \xi))^{n}}{n !}+\sum_{n=0}^{\infty} \frac{\theta^{n}(F(x ; \xi))^{n+1}}{n !}\right\} \mathrm{d} x \\
\mu_{r}^{\prime}=\frac{1}{\left(e^{\theta}+1\right)}\left\{\begin{array}{c}
2 \int_{\Omega} x^{r} f(x ; \xi) F(x ; \xi) \mathrm{d} x+2 \sum_{n=0}^{\infty} \frac{\theta^{n}}{n !} \int_{\Omega} x^{r} f(x ; \xi)(F(x ; \xi))^{n+1} \mathrm{~d} x \\
+\sum_{n=0}^{\infty} \frac{\theta^{n}}{n !} \int_{\Omega} x^{r} f(x ; \xi)(F(x ; \xi))^{n+2} \mathrm{~d} x
\end{array}\right\} .
\end{gathered}
$$

Let

$$
\begin{aligned}
\eta_{r} & =\int_{\Omega} x^{r} f(x ; \xi) F(x ; \xi) \mathrm{d} x, \\
\eta_{r, n+1} & =\int_{\Omega} x^{r} f(x ; \xi)(F(x ; \xi))^{n+1} \mathrm{~d} x, \\
\eta_{r, n+2} & =\int_{\Omega} x^{r} f(x ; \xi)(F(x ; \xi))^{n+2} \mathrm{~d} x .
\end{aligned}
$$

Putting all these values in equation (12), we get

$$
\mu_{r}^{\prime}=2 \eta_{r}+2 \sum_{n=0}^{\infty} \frac{\theta^{n}}{n !} \eta_{r, n+1}+\sum_{n=0}^{\infty} \frac{\theta^{n}}{n !} \eta_{r, n+2}
$$

4.2. Moment Generating Function. The moment generating function (mgf) of random variable $X$ is given by the following expression:

$$
\begin{aligned}
& M_{x}(t)=\int_{\Omega} e^{t x} g(x ; \theta, \xi) \mathrm{d} x, \\
& M_{x}(t)=\int_{\Omega} \sum_{n=0}^{\infty} \frac{t^{r} x^{r}}{r !} g(x ; \theta, \xi) \mathrm{d} x, \\
& M_{x}(t)=\sum_{r=0}^{\infty} \frac{t^{r}}{r !} \int_{\Omega} x^{r} g(x ; \theta, \xi) \mathrm{d} x, \\
& M_{x}(t)=\sum_{r=0}^{\infty} \frac{t^{r}}{r !} \mu_{r}^{\prime} .
\end{aligned}
$$

4.3. Quantile Function. The quantile function is the best approach to identify the distribution of a variable. The closed-form expression of the quantile function of the NEE class makes it possible to conduct a simulation study very easily from the proposed family. The quantile function of the
NEE class of distributions is provided by the expression given as follows:

$$
x_{q}=Q(u)=G^{-1}(u)=F^{-1}(t),
$$

where " $t$ " is the solution of $\log \left(e^{\theta t}+1\right)+2 \log t-\log \left(e^{\theta}+1\right), \quad U$ has the uniform distribution.

\section{Estimation of Parameters and Monte Carlo Simulation Study}

Here, we will discuss the estimation of unknown parameters, and to support these estimates, a comprehensive simulation study is provided. Moreover, the results of the simulation study are exhibited via the graphical method.

5.1. Maximum Likelihood Estimation. In this portion, we continue by illustrating the estimates of parameters of the NEE class of distributions via the maximum likelihood (ML) estimation method. Several methods were introduced in the literature to estimate the model's parameters, but the ML estimation method is the most widely used method. The ML estimators fulfill the required properties. Let $X_{i}$ be a random variable distributed as in equation (4) and $X_{1}, X_{2}, \ldots, X_{k}$ be the observed values collected from the NEE class having parameters $(\theta, \xi)$. Then, the expression for the log-likelihood function is given by

$$
\begin{aligned}
\log L(x ; \theta, \xi)= & \sum_{i=1}^{n} \log f(x ; \xi)+\sum_{i=1}^{n} \log F(x ; \xi)+ \\
& \sum_{i=1}^{n} \log \left[2+2 e^{\theta F(x ; \xi)}+\theta F(x ; \xi) e^{\theta F(x ; \xi)}\right]-n\left(e^{\theta}+1\right) .
\end{aligned}
$$

Taking partial derivatives of the log-likelihood function, we get 


$$
\begin{aligned}
\frac{\partial \log L}{\partial \theta}= & \sum_{i=1}^{n} \frac{\left[\left\{2 e^{\theta F(x ; \xi)} F(x ; \xi)\right\}+F(x ; \xi)\left\{\theta e^{\theta F(x ; \xi)} F(x ; \xi)+e^{\theta F(x ; \xi)}\right\}\right]}{2+2 e^{\theta F(x ; \xi)}+\theta F(x ; \xi) e^{\theta F(x ; \xi)}}-n e^{\theta}, \\
\frac{\partial \log L}{\partial \xi}= & \sum_{i=0}^{n} \frac{\partial f(x ; \xi) / \partial \xi}{f(x ; \xi)}+\sum_{i=0}^{n} \frac{\partial F(x ; \xi) / \partial \xi}{F(x ; \xi)} \\
& +\sum_{i=0}^{n} \frac{\left[\left\{2 e^{\theta F(x ; \xi)} \partial F(x ; \xi) / \partial \xi\right\}+\left\{\theta\left(F(x ; \xi) e^{\theta F(x ; \xi)} \partial F(x ; \xi) / \partial \xi+e^{\theta F(x ; \xi)} \partial F(x ; \xi) / \partial \xi\right)\right\}\right]}{2+2 e^{\theta F(x ; \xi)}+\theta F(x ; \xi) e^{\theta F(x ; \xi)}} .
\end{aligned}
$$

By equating to zero and solving numerically, the expressions $(\partial / \partial \theta) \log L(x ; \theta, \xi)$ and $(\partial / \partial \xi) \log L(x ; \theta, \xi)$ will provide the ML estimates for the unknown model parameters.

5.2. Simulation Study. A comprehensive simulation study was performed to evaluate the efficiency of the maximum likelihood estimates. The precision of the maximum likelihood estimates is studied through bias, absolute bias, and the mean square error (MSE) for taking different samples by considering different parameter values. The features of the simulation study are as follows:

(i) We performed 1000 repetitions from NEE-W distribution to quantify the bias and MSE, by taking samples of sizes $n=25,50, \ldots, 1000$.

(ii) The initial values of the model parameters are chosen as indicated in Tables 1-2.

(iii) To calculate bias and MSE, expressions $\operatorname{Bias}(\widehat{\varphi})=(1 / 1000) \sum_{i=1}^{1000}(\widehat{\varphi}-\varphi)$ and$\operatorname{MSE}(\widehat{\varphi})=(1 / 1000) \sum_{i=1}^{1000}(\widehat{\varphi}-\varphi)^{2}$ are used, respectively.

Where $\varphi=(\alpha, \lambda, \theta)$. The numerical values of the study are exhibited via Tables 1, 2, while Figures 3 and 4 provide simulation results in graphical form for two different sets of parameter values (set-1: $\alpha=1.0, \lambda=0.8, \theta=1.1$, set 2 : $\alpha=1.0, \lambda=0.7, \theta=0.6$ ). From the plots, it is obvious that the estimates tend to be stable as $n$ increases. Similarly, the estimated MSEs approach towards zero as sample size increases. Moreover, the absolute and estimated biases decrease as the sample size increases. The numerical results presented in the plots reveal the consistency of the MLEs.

\subsubsection{Simulation Set 2 .}

\section{Applications to COVID-19 Data}

This section presents the applicability and goodness of fit of the proposed model. Here we considered three COVID-19 datasets describing the mortality rates of different countries. The proposed model is compared with three well-known existing probability models, named alpha power transformed Weibull (APTW), Kumaraswamy Weibull $(\mathrm{Ku}-\mathrm{W})$, and exponentiated Weibull (EW) models.

The numerical estimates of the unknown model parameters are obtained via $\mathrm{R}$ software using the package
"ModelAdequacy". Different analytical tools are employed in order to assess the model's adequacy. These measures include Kolmogorov-Smirnov (K. S), Cramer-von Mises (C. M), Anderson-Darling (A. D), Akaike information criterion (AIC), Hannan-Quinn (HQIC), Bayesian information criterion (BIC), and consistent Akaike information criterion (CAIC). The numerical estimates of the aforementioned measures are exhibited for each dataset separately, which demonstrates that the proposed model is the best fit and the best candidate model among the other competing models.

6.1. Data 1: Canada Data. The first datasets contain 36 observations showing the mortality rates of COVID-19 patients in Canada. This data can be checked via the link [https://covid19.who.int/], this dataset was used by [26]. These observations are presented as follows:

$3.1091,3.3825,2.8636,3.2218,4.2781,4.2202,2.1901$, 2.4141, 1.9048, 2.9078, 3.6426, 3.2110, 3.6346, 2.7957, 1.5157, 2.6029, 3.3592, 2.8349, 3.1348, 2.5261, 1.5806, 2.7704, 3.8594, $4.0480,4.1685,3.1444,3.2135,2.4946,3.5146,4.9274,3.3769$, 6.8686, 3.0914, 4.9378, 3.1091, 3.2823.

Table 3 exhibits the ML estimates and associated standard errors, enclosed in parenthesis, for the proposed model using the Canadian data. Moreover, the numerical results of the analytical measures are presented in Table 4, demonstrating that the new model is best fitted and gives satisfactory results among the four competing probability distributions.

The plots of the joint CDF and pdf given in Figure 5, illustrate that the NEE-W model is better fitted than the other compared distributions, while the PP plot for normality and survival plots are presented in Figure 6, using data 1 .

6.2. Data 2: The Netherlands Data. The second dataset considered by [27] comprises the 30 observations of the COVID-19 patients, recorded in the Netherlands from the period of 31 March to 30 April 2020. The observations of the second data set are provided as follows:

$14.918,7.498,6.940,10.656,2.857,2.254,12.274,10.289$, $10.832,7.099,3.461,3.647,5.928,13.211,7.968,7.584,5.307$, 5.048, 5.431, 5.555, 6.027, 4.097, 3.611, 4.960, 4.462, 3.883, $1.974,1.273,1.416,4.235$.

The ML method of estimation is used to find out the numerical estimates of the unknown model parameters 
TABLE 1: The numerical results of the set-1 simulation study for the NEE-W model.

\begin{tabular}{|c|c|c|c|c|}
\hline \multirow{2}{*}{$N$} & \multicolumn{4}{|c|}{$\alpha=1.0, \lambda=0.8, \theta=1.1$} \\
\hline & Parameters & MLEs & MSEs & Biases \\
\hline \multirow{3}{*}{25} & $\alpha$ & 1.688149 & 1.1863495209 & 0.6881494182 \\
\hline & $\lambda$ & 1.0760577 & $1.222714 e-01$ & 0.2760576871 \\
\hline & $\theta$ & 4.3292 & 12.59388 & 3.2292 \\
\hline \multirow{3}{*}{50} & $\alpha$ & 1.482220 & 0.5720283252 & 0.4822204495 \\
\hline & $\lambda$ & 1.0248372 & $8.870046 e-02$ & 0.2248372074 \\
\hline & $\theta$ & 3.7481 & 10.32759 & 2.6481 \\
\hline \multirow{3}{*}{100} & $\alpha$ & 1.291446 & 0.2429927881 & 0.2914460436 \\
\hline & $\lambda$ & 0.9487059 & $5.145887 e-02$ & 0.1487058741 \\
\hline & $\theta$ & 2.9447 & 7.19433 & 1.8447 \\
\hline \multirow{3}{*}{200} & $\alpha$ & 1.132705 & 0.0875703779 & 0.1327048989 \\
\hline & $\lambda$ & 0.8716795 & $2.321303 e-02$ & 0.0716794823 \\
\hline & $\theta$ & 2.0048 & 3.52872 & 0.9048 \\
\hline \multirow{3}{*}{400} & $\alpha$ & 1.018154 & 0.0098673818 & 0.0181542892 \\
\hline & $\lambda$ & 0.8106912 & $3.180431 e-03$ & 0.0106912429 \\
\hline & $\theta$ & 1.2443 & 0.56277 & 0.1443 \\
\hline \multirow{3}{*}{600} & $\alpha$ & 1.004494 & 0.0025796913 & 0.0044940585 \\
\hline & $\lambda$ & 0.8024480 & $7.532611 e-04$ & 0.0024479757 \\
\hline & $\theta$ & 1.1312 & 0.12168 & 0.0312 \\
\hline \multirow{3}{*}{800} & $\alpha$ & 1.001256 & 0.0007950773 & 0.0012561500 \\
\hline & $\lambda$ & 0.8006441 & $2.079034 e-04$ & 0.0006440781 \\
\hline & $\theta$ & 1.1078 & 0.03042 & 0.0078 \\
\hline \multirow{3}{*}{1000} & $\alpha$ & 1.000565 & 0.0003186683 & 0.0005645071 \\
\hline & $\lambda$ & 0.8003251 & $1.056993 e-04$ & 0.0003251144 \\
\hline & $\theta$ & 1.1039 & 0.01521 & 0.0039 \\
\hline
\end{tabular}

TABle 2: The numerical results of the MLEs, MSEs, and biases for the NEE-W model using different values of parameters.

\begin{tabular}{|c|c|c|c|c|}
\hline \multirow{2}{*}{$N$} & \multicolumn{4}{|c|}{$\alpha=1, \lambda=0.7, \theta=0.6$} \\
\hline & Parameters & MLEs & MSEs & Biases \\
\hline \multirow{3}{*}{25} & $\alpha$ & 0.3396730 & 0.5419019568 & -0.6603270020 \\
\hline & $\lambda$ & 0.8119746 & $3.172569 e-02$ & 0.1119745968 \\
\hline & $\theta$ & 4.415583 & 16.80649 & 3.815583 \\
\hline \multirow{3}{*}{50} & $\alpha$ & 0.4062511 & 0.4678004494 & -0.5937488691 \\
\hline & $\lambda$ & 0.7836855 & $1.569647 e-02$ & 0.0836854504 \\
\hline & $\theta$ & 3.956601 & 14.77204 & 3.356601 \\
\hline \multirow{3}{*}{100} & $\alpha$ & 0.5863305 & 0.3241790787 & -0.4136694671 \\
\hline & $\lambda$ & 0.7564958 & $8.092500 e-03$ & 0.0564957897 \\
\hline & $\theta$ & 2.923200 & 10.22208 & 2.323200 \\
\hline \multirow{3}{*}{200} & $\alpha$ & 0.8074348 & 0.1507560442 & -0.1925651699 \\
\hline & $\lambda$ & 0.7259528 & $3.253839 e-03$ & 0.0259528461 \\
\hline & $\theta$ & 1.682400 & 4.76256 & 1.082400 \\
\hline \multirow{3}{*}{400} & $\alpha$ & 0.7061294 & 0.0349877432 & -0.0446563129 \\
\hline & $\lambda$ & 0.9553437 & $7.262195 e-04$ & 0.0061293605 \\
\hline & $\theta$ & 0.850800 & 1.10352 & 0.250800 \\
\hline \multirow{3}{*}{600} & $\alpha$ & 0.9882905 & 0.0091412466 & -0.0117094601 \\
\hline & $\lambda$ & 0.7014644 & $1.476944 e-04$ & 0.0014643526 \\
\hline & $\theta$ & 0.666000 & 0.29040 & 0.066000 \\
\hline \multirow{3}{*}{800} & $\alpha$ & 0.9968609 & 0.0024635519 & -0.0031391276 \\
\hline & $\lambda$ & 0.7003741 & $3.541752 e-05$ & 0.0003740722 \\
\hline & $\theta$ & 0.617600 & 0.07744 & 0.017600 \\
\hline \multirow{3}{*}{1000} & $\alpha$ & 1.0000000 & 0.0000000000 & 0.0000000000 \\
\hline & $\lambda$ & 0.7000000 & $0.000000 e+00$ & 0.0000000000 \\
\hline & $\theta$ & 0.600000 & 0.00000 & 0.000000 \\
\hline
\end{tabular}






(a)

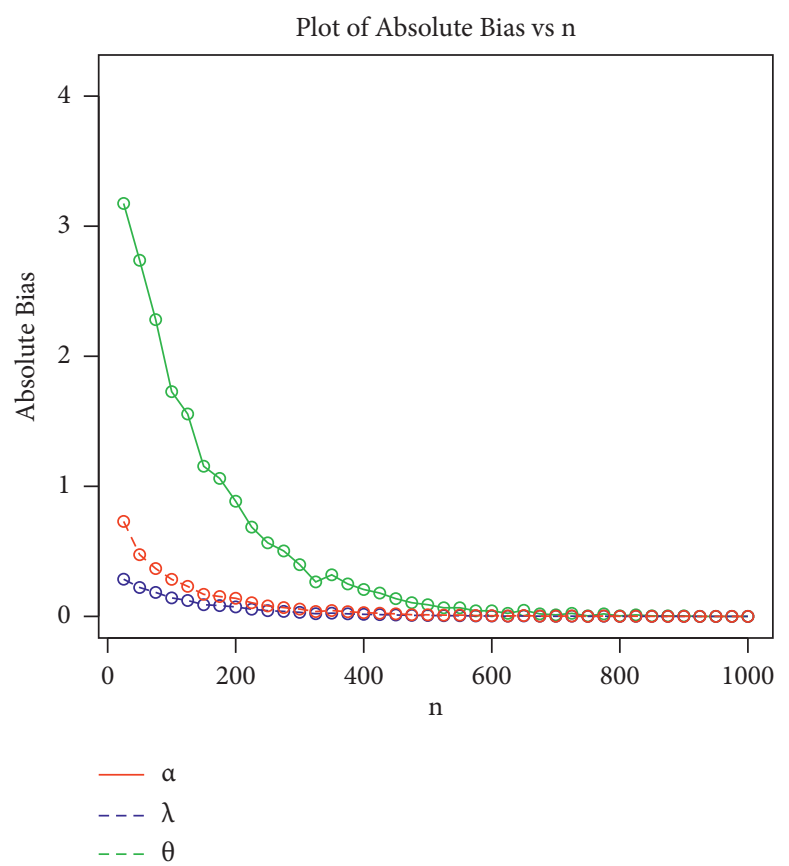

(c)

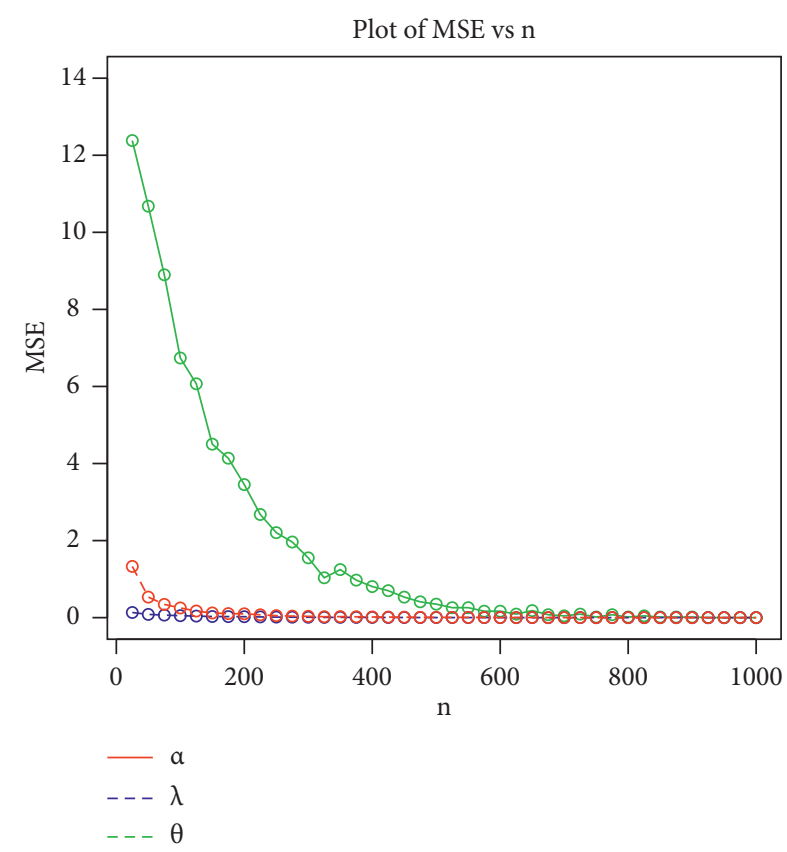

(b)

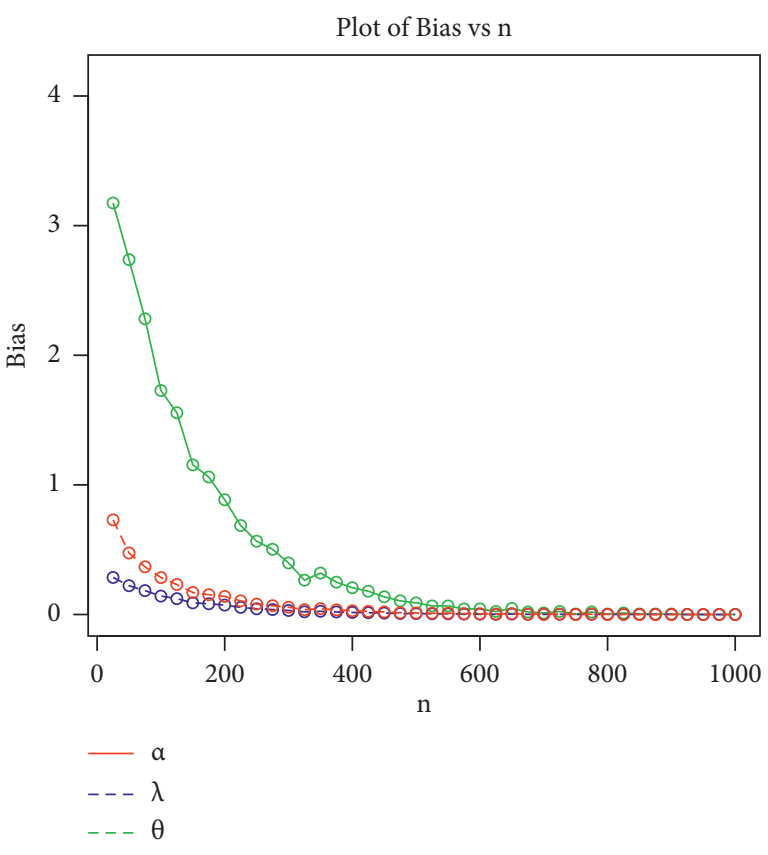

(d)

Figure 3: Different plots of the set-1 simulation results for the NEE-W distribution.

along with their corresponding standard errors and is provided in Table 5. Furthermore, the results of different statistics are obtained through the $\mathrm{R}$ language using the "ModelAdequacy" package. The results presented in Table 6, give evidence as the proposed model is the best candidate model as compared to the three existing models.

Figure 7 provides the joint CDF and pdf plots for NEE$\mathrm{W}$ and three competing distributions using the Netherlands dataset. The plots presented in Figure 7 explain that the NEE-W distribution is a better fit than the other models.
While Figure 8 presents the PP and Kaplan-Meier survival plots for the NEE-W model, showing the best fitting of the NEE-W model for the Netherlands data.

6.3. Data 3: Mexico Data. The third set of data holds 108 observations, demonstrating the COVID-19 mortality rates in Mexico. This dataset was used by [27]. The duration of recording this data is from 4 March to 20 July 2020 . These observations are presented as follows: 


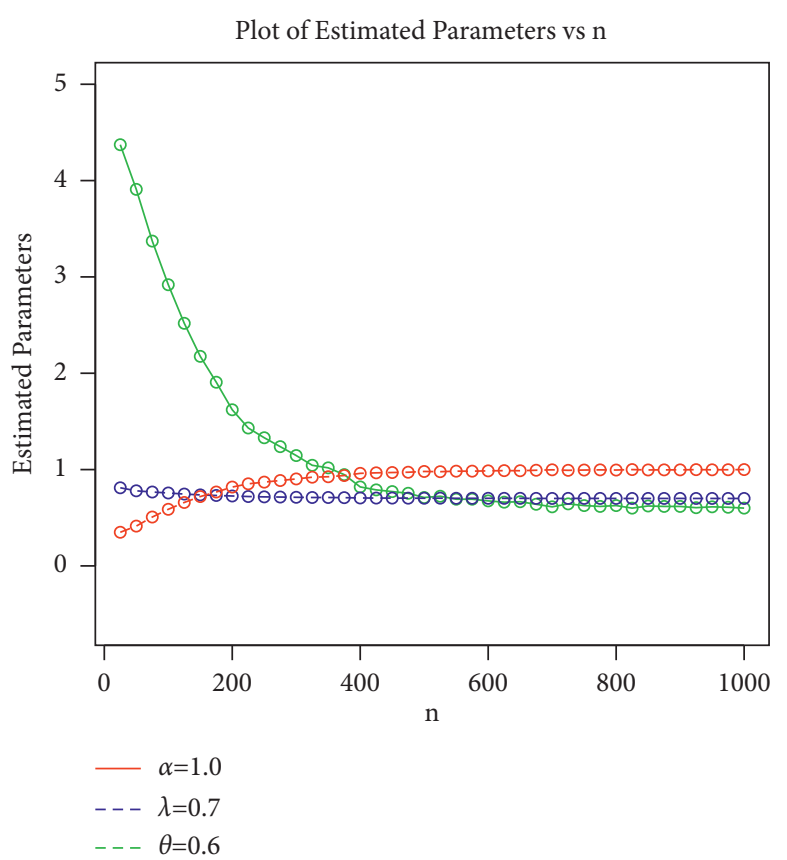

(a)

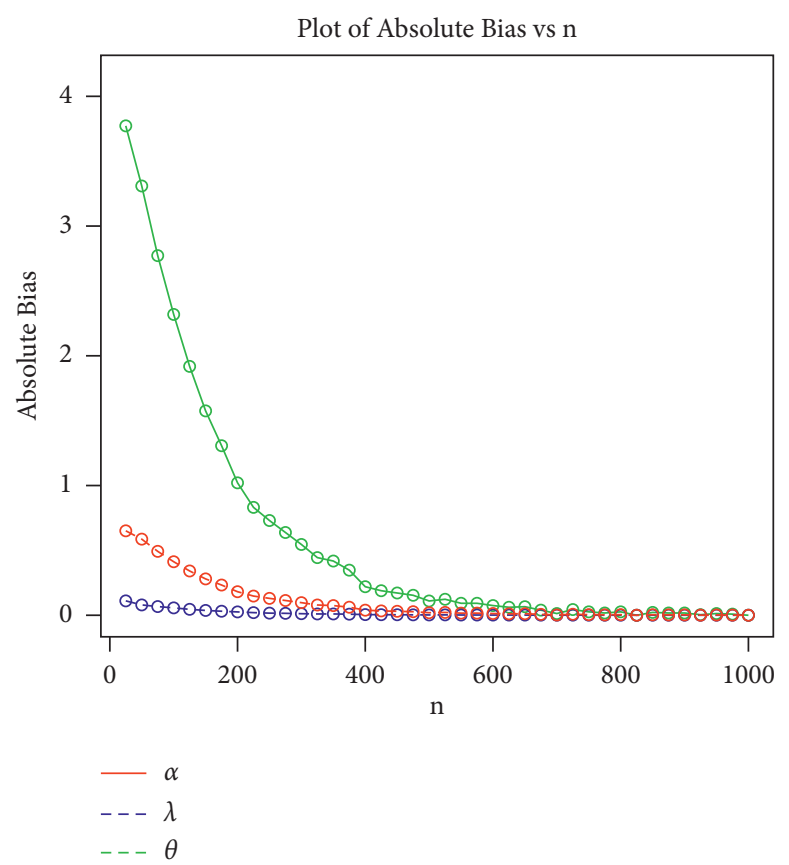

(c)



(b)

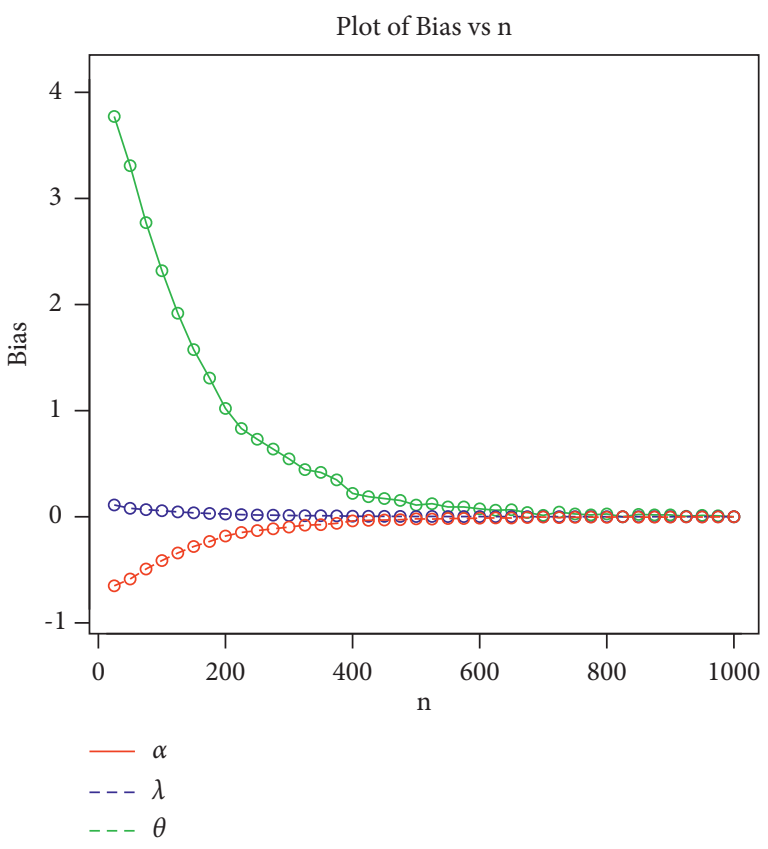

(d)

FIGURE 4: The plots of estimated parameters, MSEs, absolute bias, and bias for the NEE-W model using the results of set 2.

TABLE 3: MLE's and their standard errors for the proposed, APTW, Ku-W, and EFW models.

\begin{tabular}{lcccccccc}
\hline Distributions & $\hat{\alpha}$ & $\widehat{\theta}$ & $\widehat{\lambda}$ & $\widehat{\beta}$ & $\widehat{c}$ & $\widehat{\kappa}$ & $\hat{a}$ & \\
\hline NEE-W & $0.064(0.026)$ & $0.007(0.336)$ & $2.515(0.293)$ & & & & & \\
APTW & $1.022(\mathrm{NaN})$ & $3.291(\mathrm{NaN})$ & & $0.014(\mathrm{NaN})$ & & & & \\
Ku-W & & & & & $0.701(0.310)$ & $0.133(0.023)$ & $2.396(0.002)$ & $0.353(0.002)$ \\
EFW & $0.228(0.013)$ & & $0.168(0.059)$ & $1.220(0.609)$ & & & & \\
\hline
\end{tabular}


TABLE 4: The numerical results of Canadian COVID-19 data for the proposed and competing models.

\begin{tabular}{|c|c|c|c|c|c|c|c|}
\hline Distributions & K.S & C-M & A-D & AIC & BIC & CIAC & HQIC \\
\hline NEE-W & 0.135 & 0.122 & 0.699 & 104.66 & 109.41 & 105.41 & 106.32 \\
\hline APTW & 0.148 & 0.172 & 0.989 & 108.96 & 113.71 & 109.71 & 110.62 \\
\hline $\mathrm{Ku}-\mathrm{W}$ & 0.242 & 0.140 & 0.801 & 119.43 & 125.77 & 120.72 & 121.64 \\
\hline EFW & 0.271 & 0.625 & 3.584 & 146.33 & 151.08 & 147.08 & 147.99 \\
\hline
\end{tabular}

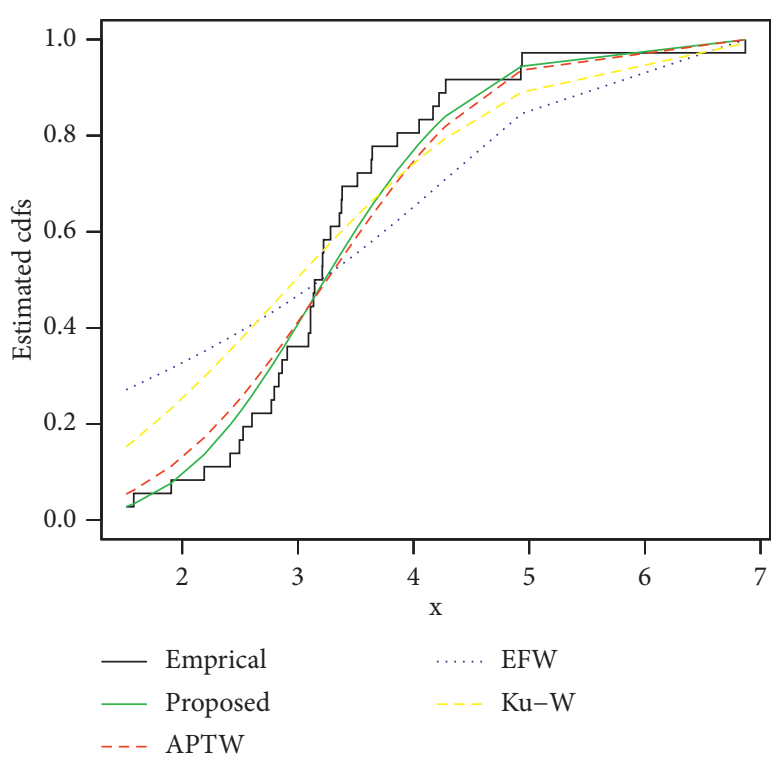

(a)

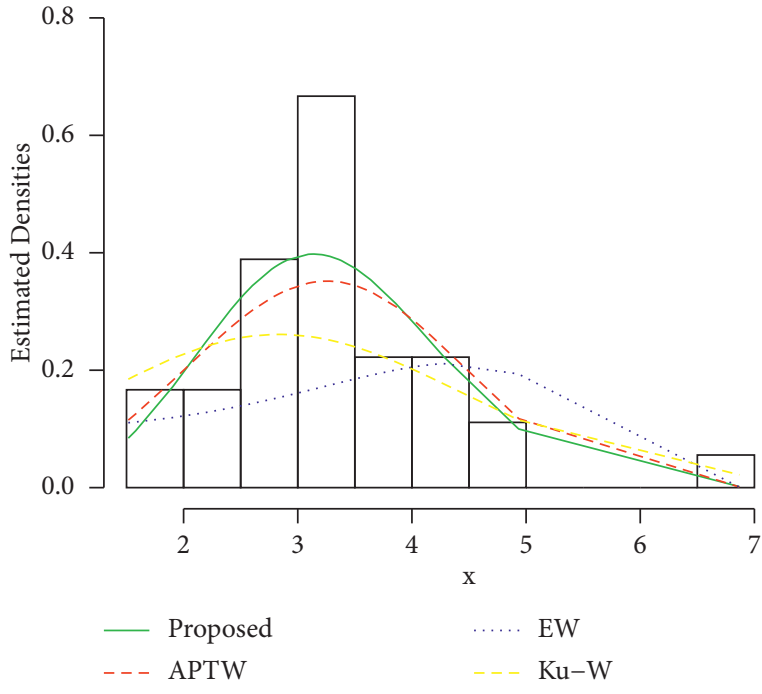

(b)

FIGURE 5: Fitted plots of joint CDF and pdf for the proposed and four competing distributions.

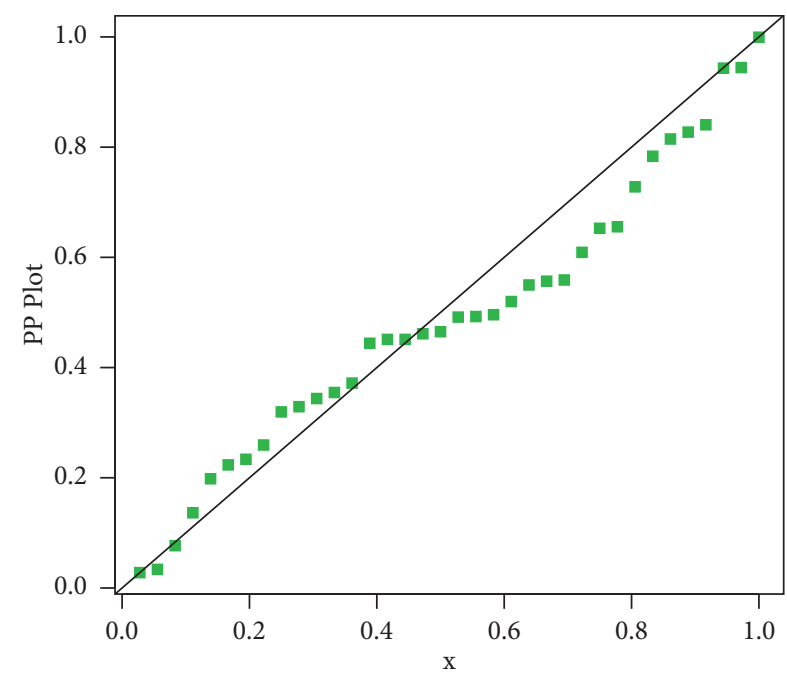

(a)

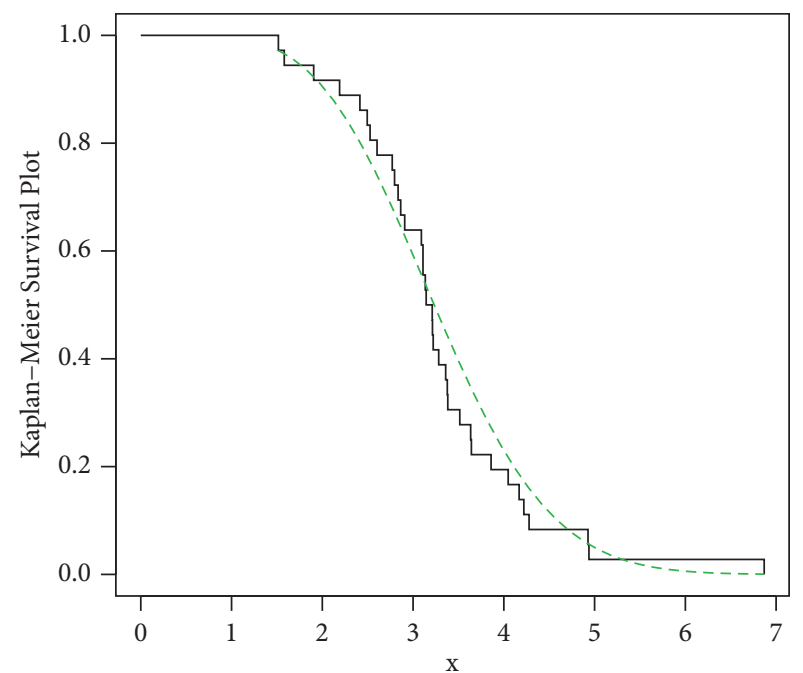

(b)

FIgURE 6: PP and Kaplan-Meier survival plots for the proposed model using COVID-19 data collected in Canada.

$8.826,6.105,9.391,14.962,10.383,7.267,13.220,16.498$, $11.665,6.015,10.855,6.122,6.656,3.440,5.854,10.685$, $10.035,5.242,4.344,5.143,7.630,14.604,7.903,6.370,3.537$, $6.327,4.730,3.215,9.284,12.878,8.813,10.043,7.260,5.985$, $6.412,3.395,4.424,9.935,7.840,9.550,3.499,3.751,6.968$,
$3.286,10.158,8.108,6.697,7.151,6.560,2.077,3.778,2.988$, $3.336,6.814,8.325,7.854,8.551,3.228,7.486,6.625,6.140$, $4.909,4.661,5.392,12.042,8.696,1.815,3.327,5.406,6.182$, $1.041,1.800,4.949,4.089,3.359,2.070,3.298,5.317,5.442$, $4.557,4.292,2.500,6.535,4.648,4.697,5.459,4.120,3.922$, 
TABle 5: Providing the numerical estimates and standard errors for the proposed and four compared models.

\begin{tabular}{|c|c|c|c|c|c|c|c|c|}
\hline Distributions & $\widehat{\alpha}$ & $\widehat{\theta}$ & $\widehat{\lambda}$ & $\widehat{\beta}$ & $\widehat{c}$ & $\widehat{\kappa}$ & $\widehat{a}$ & $\widehat{b}$ \\
\hline NEE-W & $0.1280(0.050)$ & $-0.006(0.365)$ & $1.319(0.181)$ & & & & & \\
\hline APTW & $0.428(0.866)$ & $1.807(0.434)$ & & $0.024(0.035)$ & & & & \\
\hline $\mathrm{Ku}-\mathrm{W}$ & & & & & $0.249(\mathrm{NaN})$ & $0.092(\mathrm{NaN})$ & $1.526(\mathrm{NaN})$ & $0.563(\mathrm{NaN})$ \\
\hline EFW & $0.089(0.008)$ & & $0.194(0.061)$ & $1.877(0.800)$ & & & & \\
\hline
\end{tabular}

TABLE 6: The results of the goodness of fit and other analytical measures for the Netherland's data.

\begin{tabular}{lccccccc}
\hline Distributions & K.S & C-M & A-D & AIC & BIC & CIAC & HQIC \\
\hline NEE-W & 0.079 & 0.027 & 0.193 & 159.39 & 163.59 & 160.31 & 160.73 \\
APTW & 0.114 & 0.033 & 0.219 & 160.37 & 164.57 & 161.29 & 161.71 \\
Ku-W & 0.209 & 0.058 & 0.357 & 169.61 & 175.22 & 171.21 \\
EFW & 0.221 & 0.324 & 1.784 & 179.78 & 183.98 & 180.70 & 171.40 \\
\hline
\end{tabular}

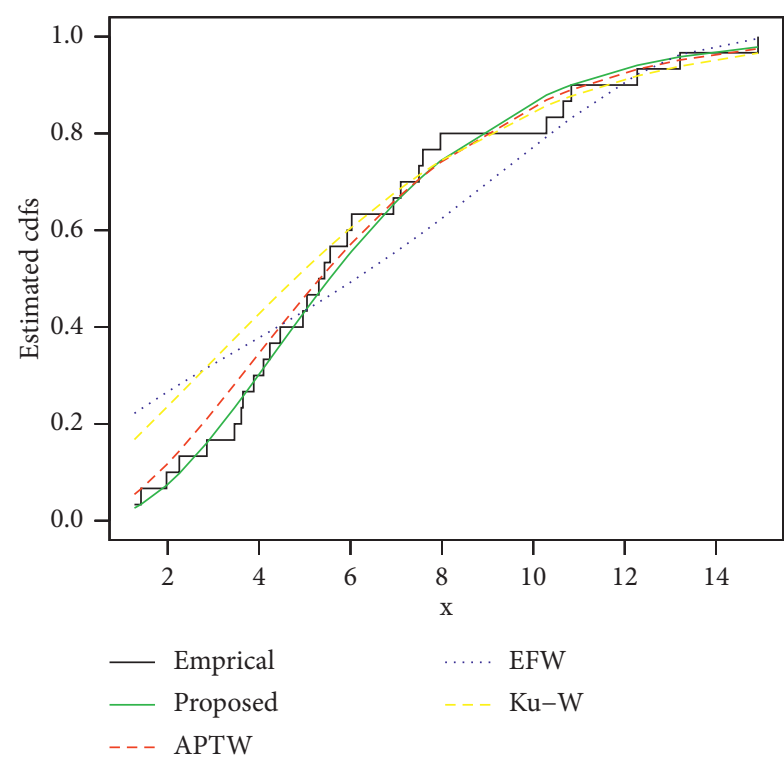

(a)

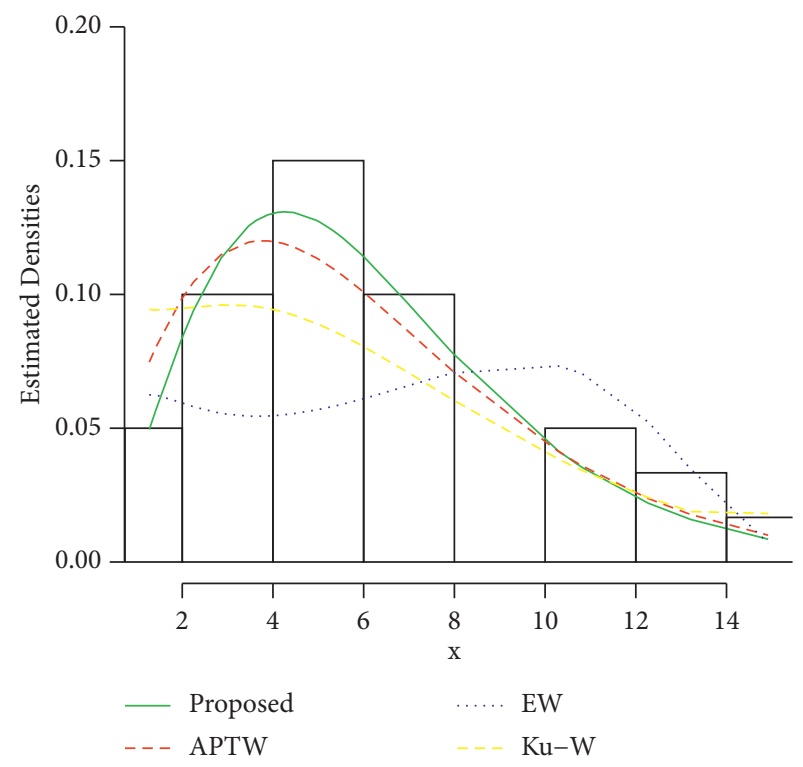

(b)

FIgURe 7: Fitted plots of joint CDF and pdf for proposed and competing models using COVID-19 data collected in the Netherlands.

$3.219,1.402,2.438,3.257,3.632,3.233,3.027,2.352,1.205$, $3.218,2.926,2.601,2.065,3.029,2.058,2.326,2.506,1.923$.

The results of the ML estimates and the analytical statistics for the third dataset are provided in Tables 7 and 8, respectively, showing the best fitting of the proposed distribution. Hence, for all three datasets, the proposed model proves itself to be the best-fitted model.

The plots of the joint CDF and pdf presented in Figure 9 explain the best fitting of the NEE-W and other compared distributions using Mexico data. The plots presented in Figure 9 clearly indicate that the NEE-W model is better fitted than the other models. While, the best-fit PP and Kaplan-Meier survival plots presented in Figure 10 make it evident that NEE-W is the best candidate model for such types of datasets.

\section{Limitations of the Proposed Model}

Although the suggested model is the best candidate model among the competing models as shown by the results of different analytical measures, there are still some limitations to our proposed model. The limitations of the proposed model are given as follows:

(i) The MLE's are not in closed form, so we need to use software to compute the numerical values of the estimates.

(ii) The proposed model is a continuous probability model and it can be used to model continuous datasets. It is not applicable to discrete data.

(iii) The CDF of the proposed model is not in simple form; therefore, for the generation of random numbers, we need to use the software.

\section{Future Research Direction}

We have proposed a NEE class of distributions in this paper. For future research directions, one can use exponentiated and Kumaraswamy techniques in order to bring more 


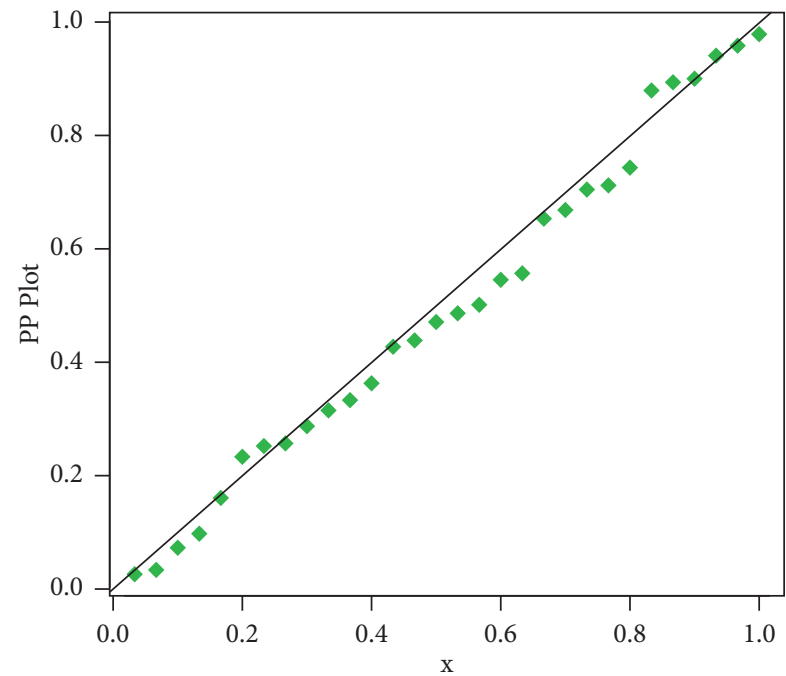

(a)

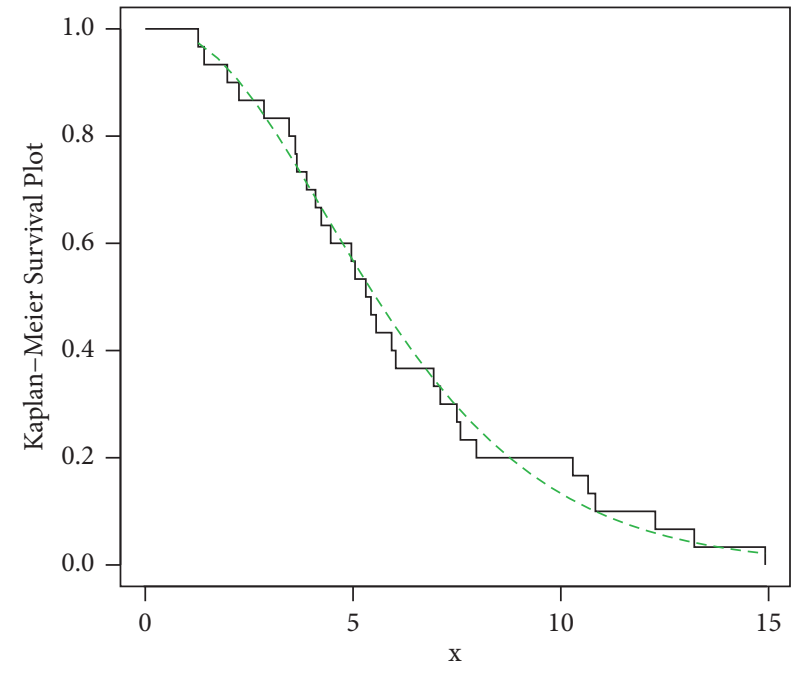

(b)

Figure 8: Using the Netherlands COVID-19 data and fitting the PP and Kaplan-Meier survival plots.

TABLe 7: The numerical values of the proposed and compared distributions using the ML technique.

\begin{tabular}{|c|c|c|c|c|c|c|c|c|}
\hline Distributions & $\widehat{\alpha}$ & $\widehat{\theta}$ & $\hat{\lambda}$ & $\widehat{\beta}$ & $\widehat{c}$ & $\widehat{\kappa}$ & $\widehat{a}$ & $\widehat{b}$ \\
\hline NEE-W & $0.132(0.027)$ & $-0.010(0.096)$ & $1.346(0.192)$ & & & & & \\
\hline APTW & $0.102(0.110)$ & $0.007(0.003)$ & & $2.217(0.140)$ & & & & \\
\hline $\mathrm{Ku}-\mathrm{W}$ & & & & & $0.558(0.040)$ & $0.131(0.012)$ & $1.748(0.002)$ & $0.266(0.002)$ \\
\hline EFW & $0.087(0.004)$ & & $0.230(0.035)$ & $1.997(0.408)$ & & & & \\
\hline
\end{tabular}

TABLE 8: Statistics of the analytical measures using COVID-19 data of Mexico.

\begin{tabular}{|c|c|c|c|c|c|c|c|}
\hline Distributions & K.S & $\mathrm{C}-\mathrm{M}$ & A-D & AIC & $\mathrm{BIC}$ & CIAC & HQIC \\
\hline NEE-W & 0.074 & 0.070 & 0.419 & 539.64 & 547.69 & 539.87 & 542.90 \\
\hline APTW & 0.076 & 0.082 & 0.495 & 540.76 & 548.80 & 540.99 & 544.02 \\
\hline $\mathrm{Ku}-\mathrm{W}$ & 0.117 & 0.137 & 0.882 & 557.2 & 567.92 & 557.58 & 561.55 \\
\hline EFW & 0.259 & 0.891 & 5.358 & 630.27 & 638.32 & 630.50 & 633.54 \\
\hline
\end{tabular}

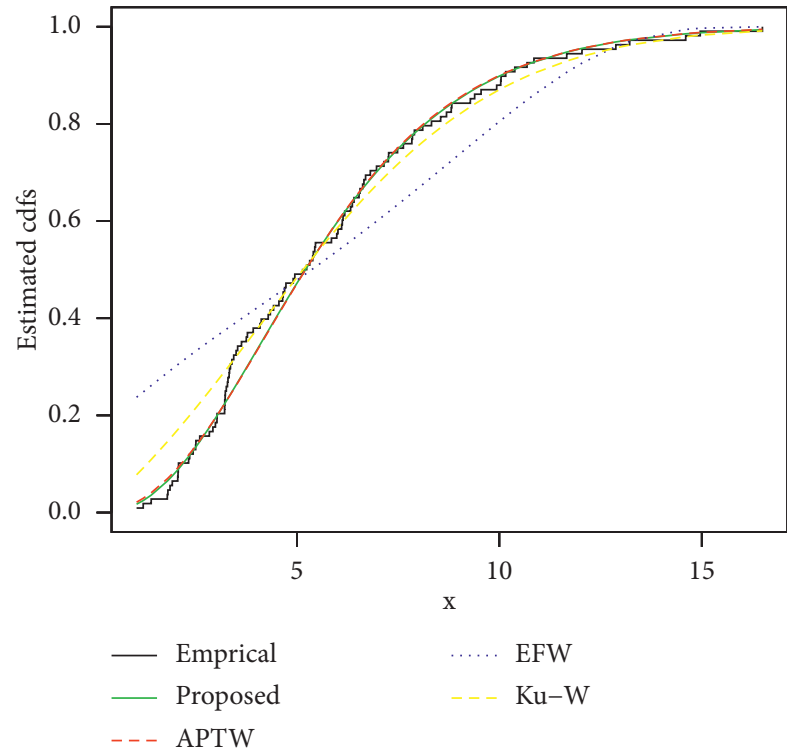

(a)

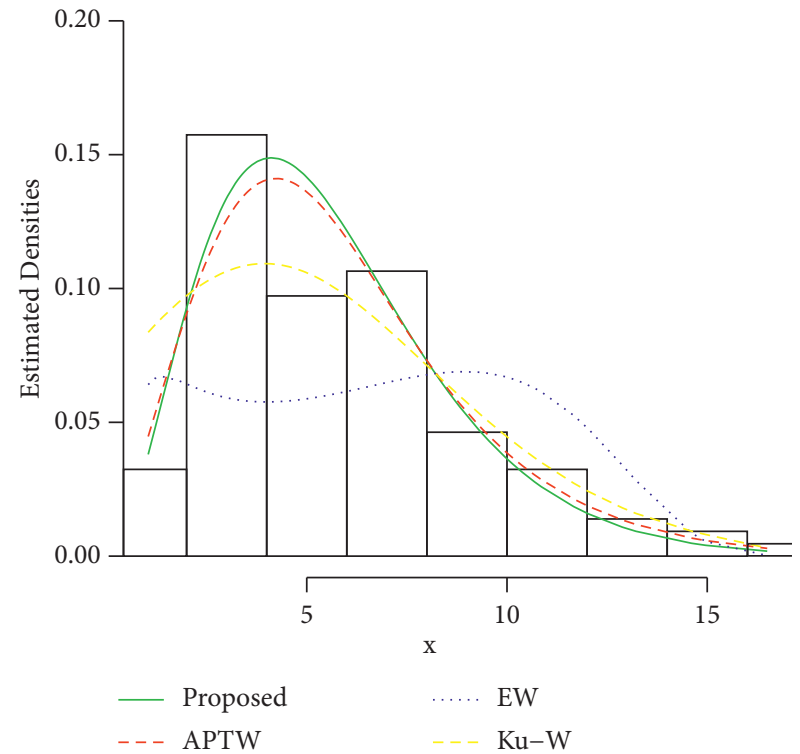

(b)

FIGURE 9: Showing the joint plots of CDF and pdf for the proposed and competing models using the COVID-19 data in Mexico. 


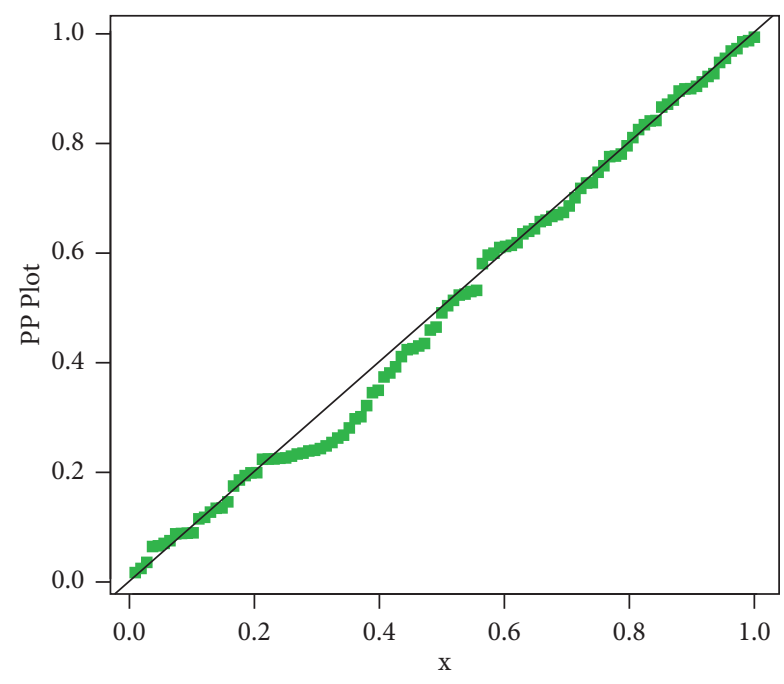

(a)



(b)

FIgURE 10: Best-fitted PP and Kaplan-Meier Survival plots for the proposed distribution using the third dataset.

flexibility in the NEE class of distributions. Suppose $X$ is a random variable having the NEE family, then the exponentiated form of the NEE class can be expressed by the following $\mathrm{CDF}$ as follows:

$\kappa(x ; \alpha, \zeta)=\left(\frac{\left(e^{\theta F(x ; \xi)}+1\right)(F(x ; \xi))^{2}}{e^{\theta}+1}\right)^{\alpha}, \quad x \geq 0, \alpha, \zeta>0$.

Similarly, we can also use the Kumaraswamy technique as suggested by [24-26] in order to modify our suggested family of distributions and to present a more parsimonious version of the proposed family. Suppose $X$ is distributed with the NEE class of distributions, then the CDF of the new suggested model will be as follows:

$$
\kappa(x ; \alpha, b, \zeta)=1-\left(1-\left(\frac{\left(e^{\theta F(x ; \xi)}+1\right)(F(x ; \xi))^{2}}{e^{\theta}+1}\right)^{\alpha}\right)^{b} .
$$

\section{Conclusion}

The probability models have shown great significance and got the attention of the researchers to model the data using these probability models, especially in survival analysis. COVID-19 is the most dangerous virus that has badly affected the daily life routines of every person in the world. It is the responsibility of the government and other agencies to provide a description of the data under consideration in order to obtain good estimates of the parameters. In this article, we suggest a new parsimonious, flexible, and prominent family of continuous distribution by adding a single extra parameter to the existing models called, the novel extended exponentiated class of distributions. The most attractive and flexible nature of the new family predominates over the more appealing characterizations.
General equations for basic mathematical characterizations such as raw moments, quantile function, and mgf of the proposed family are obtained. The most widely used ML method is adopted for the estimation of unknown model parameters. The stability and efficiency of the estimates of the proposed model are assessed by a simulation study. The main discriminating property of the proposed model amongst the competitors is its closed form of CDF and having the capability of modeling data with monotonic and nonmonotonic failure rates. An illustrative example of the applicability of the new proposed family is presented in the form of a submodel case, called NEE-W distribution. Moreover, three biomedical datasets related to COVID-19, are considered to evaluate the performance of the NEE-W with the competent models given in the literature. We noticed that both the real-life applications and the simulation studies show that the proposed model has outperformed the other models considered in this study.

\section{Data Availability}

Data sharing does not apply to this article as no data set were generated or analyzed during the current study.

\section{Conflicts of Interest}

The authors declare that they have no conflicts of interest.

\section{References}

[1] R. Al-Aqtash, F. Famoye, and C. Lee, "On generating a new family of distributions using the logit function," Journal of Probability and Statistical Science, vol. 13, no. 1, pp. 135-152, 2015.

[2] A. Alzaatreh, F. Famoye, and C. Lee, "The gamma-normal distribution: properties and applications," Computational Statistics \& Data Analysis, vol. 69, pp. 67-80, 2014.

[3] Y. Liu, M. Ilyas, S. K. Khosa et al., "A flexible reduced logarithmic-X family of distributions with biomedical analysis," 
Computational and Mathematical Methods in Medicine, vol. 2020, Article ID 4373595, 15 pages, 2020.

[4] G. M. Cordeiro, E. M. Ortega, and D. C. Da Cunha, "The exponentiated generalized class of distributions," Journal of Data Science, vol. 11, no. 1, pp. 1-27, 2013.

[5] A. Alzaatreh, C. Lee, F. Famoye, and I. Ghosh, "The generalized Cauchy family of distributions with applications," Journal of Statistical Distributions and Applications, vol. 3, no. 1, p. 12, 2016.

[6] Z. M. Nofal, A. Z. Afify, H. M. Yousof, and G. M. Cordeiro, "The generalized transmuted-G family of distributions," Communications in Statistics-Theory and Methods, vol. 46, no. 8, pp. 4119-4136, 2017.

[7] A. S. Hassan and M. Elgarhy, "A new family of exponentiated Weibull-generated distributions," RN, vol. 55, p. 7, 2016.

[8] A. Mahdavi and D. Kundu, "A new method for generating distributions with an application to exponential distribution," Communications in Statistics-Theory and Methods, vol. 46, no. 13, pp. 6543-6557, 2017.

[9] M. Nassar, A. Alzaatreh, M. Mead, and O. Abo-Kasem, "Alpha power Weibull distribution: properties and applications," Communications in Statistics-Theory and Methods, vol. 46, no. 20, pp. 10236-10252, 2017.

[10] S. Dey, V. K. Sharma, and M. Mesfioui, "A new extension of Weibull distribution with application to lifetime data," Annals of Data Science, vol. 4, no. 1, pp. 31-61, 2017.

[11] S. Dey, I. Ghosh, and D. Kumar, "Alpha-power transformed Lindley distribution: properties and associated inference with application to earthquake data," Annals of Data Science, vol. 6 , no. 4, pp. 623-650, 2019.

[12] M. Zichuan, S. Hussain, A. Iftikhar et al., "A new extendedfamily of distributions: properties and applications," Computational and Mathematical Methods in Medicine, vol. 2020, Article ID 4650520, 13 pages, 2020.

[13] M. M. Mansour, M. Farsi, S. M. Mohamed, and E. M. Abd Elrazik, "Modeling the COVID-19 pandemic dynamics in Egypt and Saudi Arabia," Mathematics, vol. 9, no. 8, p. 827, 2021.

[14] M. Zuo, S. K. Khosa, Z. Ahmad, and Z. Almaspoor, "Comparison of COVID-19 pandemic dynamics in Asian countries with statistical modeling," Computational and Mathematical Methods in Medicine, vol. 2020, Article ID 4296806, 16 pages, 2020.

[15] J. Zhao, Z. Ahmad, Z. Almaspoor, M. El-Morshedy, and A. Afify, "Modeling COVID-9 pandemic dynamics in two Asian countries," Computers, Materials \& Continua, vol. 67, no. 1, pp. 965-977, 2021.

[16] X. Liu, Z. Ahmad, A. M. Gemeay, A. T. Abdulrahman, E. H. Hafez, and N. Khalil, "Modeling the survival times of the COVID-19 patients with a new statistical model: a case study from China," PLoS One, vol. 16, no. 7, Article ID e0254999, 2021.

[17] M. Ahsan-ul-Haq, M. Ahmed, J. Zafar, and P. L. Ramos, "Modeling of COVID-19 cases in Pakistan using lifetime probability distributions," Annals of Data Science, vol. 2021, pp. 1-12, 2021.

[18] J. Zhao, Z. Ahmad, and Z. Almaspoor, "Modeling the COVID-19 pandemic dynamics in Iran and China," Computers, Materials \& Continua, vol. 67, no. 2, pp. 2111-2122, 2021.

[19] W. Bo, Z. Ahmad, A. R. A. Alanzi, A. I. Al-Omari, E. H. Hafez, and S. F. Abdelwahab, "The current COVID-19 pandemic in China: an overview and corona data analysis," Alexandria Engineering Journal, vol. 61, no. 2, pp. 1369-1381, 2022.
[20] E. M. Almetwally, "The odd Weibull inverse topp-leone distribution with applications to COVID-19 data," Annals of Data Science, vol. 2021, pp. 1-20, 2021.

[21] W. Shaw and I. Buckley, "The alchemy of probability distributions: beyond Gram-Charlier expansions, and a skewkurtotic-normal distribution from a rank transmutation map," Research Report, vol. 2009, 2007.

[22] G. R. Aryal and C. P. Tsokos, "Transmuted Weibull distribution: a generalization of the Weibull probability distribution," European Journal of Pure and Applied Mathematics, vol. 4, no. 2, pp. 89-102, 2011.

[23] M. S. Khan and R. King, "Transmuted modified Weibull distribution: a generalization of the modified Weibull probability distribution," European Journal of Pure and Applied Mathematics, vol. 6, no. 1, pp. 66-88, 2013.

[24] F. Merovci, "Transmuted Rayleigh distribution," Austrian Journal of Statistics, vol. 42, no. 1, pp. 21-31, 2013.

[25] G. M. Cordeiro and M. De Castro, "A new family of generalized distributions," Journal of Statistical Computation and Simulation, vol. 81, no. 7, pp. 883-898, 2011.

[26] E. Almetwally, R. Alharbi, D. Alnagar, and E. Hafez, "A new inverted topp-leone distribution: applications to the COVID19 mortality rate in two different countries," Axioms, vol. 10, no. 1, p. 25, 2021.

[27] H. M. Almongy, E. M. Almetwally, H. M. Aljohani, A. S. Alghamdi, and E. H. Hafez, "A new extended Rayleigh distribution with applications of COVID-19 data," Results in Physics, vol. 23, Article ID 104012, 2021.

[28] K. Abbas, Z. Hussain, N. Rashid et al., "Bayesian estimation of gumbel type-II distribution under type-II censoring with medical applications," Computational and Mathematical Methods in Medicine, vol. 2020, Article ID 1876073, 11 pages, 2020.

[29] M. Arif, D. M. Khan, S. K. Khosa et al., "Modelling insurance losses with a new family of heavy-tailed distributions," CMCComputers Materials \& Continua, vol. 66, no. 1, pp. 537-550, 2021. 